\title{
Menadione Induces DNA Damage and Superoxide Radical Level In HEK293 Cells
}

\author{
Alireza Ghodsi Senejani ${ }^{1, ~ *, ~ J o s e p h ~ M a t t h e w ~ M a g r i n o " ~}{ }^{1}$, Amanda Marston ${ }^{1}$, Michelle Gregoire ${ }^{1}$, \\ Khoa Dang Dinh ${ }^{2}$
}

${ }^{1}$ Department of Biology and Environmental Science, University of New Haven, West Haven, USA

${ }^{2}$ Department of Mathematics, University of New Haven, West Haven, USA

\section{Email address:}

Asenejani@newhaven.edu (A. G. Senejani), joseph.magrino@umassmed.edu (J. M. Magrino), amzmarston@gmail.com (A. Marston), mgreg4@unh.newhaven.edu (M. Gregoire), kdinh@newhaven.edu (K. D. Dinh)

*Corresponding author

\section{To cite this article:}

Alireza Ghodsi Senejani, Joseph Matthew Magrino, Amanda Marston, Michelle Gregoire, Khoa Dang Dinh. Menadione Induces DNA Damage and Superoxide Radical Level In HEK293 Cells. Cell Biology. Vol. 7, No. 4, 2019, pp. 14-22. doi: 10.11648/j.cb.20190702.11

Received: November 15, 2019; Accepted: December 2, 2019; Published: December 11, 2019

\begin{abstract}
Reactive oxygen species (ROS) can damage cellular components, including mitochondrial and genomic DNA. Oxidized DNA can transgress into lethal double stranded breaks if not adequately repaired. Clinical reports of the major neurodegenerative diseases have denoted the presence of oxidized genomic DNA with no clear understanding of their role in disease progression. To date, little is known on the neuronal vulnerability and repair kinetics of oxidative damage. Here, we studied how DNA repair kinetics contributes to reduce neuronal viability in oxidative stress conditions. To induce internal oxidative stress, we exposed neuronal-like HEK293 and fibroblast cells to 2-Methyl-1, 4-napthoquinone (Menadione). We found HEK293 cells have a reduced viability in response to induced oxidative stress compared to fibroblasts. Furthermore data obtained from COMET analysis show increased level of DNA breaks and regressed DNA repair kinetics in treated cells. Our results show that HEK293 cells have a regressed repair kinetics that allows for oxidative damage to transgress into lethal forms of DNA damage. Our findings indicate that oxidative stress can play a key role in neurodegenerative diseases and alleviation of their presence could increase neuronal survival.
\end{abstract}

Keywords: Reactive Oxygen Species (ROS), Menadione, Oxidative Stress, Superoxide Radical, Neurodegenerative Diseases

\section{Introduction}

Modern medical therapies such as, vaccines and antibiotics have assisted in the extension of human longevity [1]. This has allowed for a number of age-related disorders to accumulate throughout the global community [1]. One being neurodegeneration, referring to the gradual decline of neuronal tissue that manifests into approximately 600 different neurodegenerative diseases [2-5]. Typically impacting elderly individuals, neurodegeneration reduces quality of life as it induces system deregulation over the years.

Neurodegeneration arise due to excessive necrosis or apoptosis of key neurons [6-9]. Though we still lack a complete picture of the initiating factors, genetic instability can arise from intracellular abnormalities such as protein aggregation, mitochondrial deregulation, and DNA damage $[6,10,11]$. Several studies have suggested that one potential source of genetic instability occurs from genomic insult by reactive oxygen species (ROS) $[6,10,11]$. Although our cells are equipped with various reducing agents (e.g. Glutathione, Superoxide Dismutase (SOD)), aging neurons have been shown to lose these protective mediators $[12,13]$.

On average, about 10,000 oxidized lesions occur on the genomic DNA (gDNA) per cell per day that create nontraditional Watson and Crick base pairs [14]. Interestingly, these lesions have been reported in a number of neurodegenerative clinical samples with little understanding of their biological importance [11, 15]. Given that these residues are found in $\mathrm{CpG}$ islands, $\mathrm{GC}$-rich regions, and 
promoters, it is theorized that they induce genetic instability $[11,15]$. These lesions can then transgress into single and/or double stranded breaks (SSBs or DSBs) that stimulate apoptosis mechanisms if not adequately removed [15]. Given that these lesions can be lethal, there is evidence to suggest that neurodgeneration might arise due to inadequate repair to oxdative damage.

Evidence for this theory has been reported in numerous studies by utilizing hydrogen peroxide, a known stimulate of lethal oxidative damage [16]. Unfortunately, exogenous agents, such as hydrogen peroxide, create different ROS than the endogenous sources and, don't accurately represent in vivo neurodegeneration events. To gain a precise picture, a compound such as Methyl-1, 4-napthoquinone (Menadione) can be utilized. Several studies have shown that Menadione induces Superoxide Anion (SOA) levels orchestrated by NADPH-cytochrome $\mathrm{P} 450$ reductase and mitochondrial NADH-ubiquinone of the electron transport chain [17-19]. By using Menadione, detailed repair kinetics can be obtained on how neurons respond and repair their gDNA in response to oxidative stress created from endogenous agents.

The Human Embryonic Kidney (HEK) 293 cell line was utilized to evaluate its viability and repair kinetics. HEK293 have been considered as neuron-like due to their neuro-gene and phenotypes [20]. Studies have shown that HEK293 expresses $\sim 60$ neuronal specific genes (e.g. neuro-filament, neuro-receptors, and neuron-specific metabolic enzymes) and have the capability of changing their membrane potential via voltage-gated ion channels (such as, potassium, sodium, and chloride channels) [21, 22]. Thus, HEK293 can be considered a neuron-like cell line and suitable model system to study neuroscience related diseases.

Given the correlation between nuclear oxidization and neurodegeneration, we investigated neuronal-like HEK293 cells DNA repair kinetics when exposed to endogenous ROS produced by Menadione and if the effect would be reversed when HEK293 were incubated with N-acetyl cysteine (NAC) an antioxidant, in conjunction with Menadione.

\section{Materials and Methods}

\subsection{Cell Culturing}

HEK293 and Wild Type Mouse Embryonic Fibroblasts (WT MEF) cells were a gift from Yale Medical School (New Haven, CT). Human Lung Fibroblast (WI-38) cells were provided from the Zito laboratory of the University of New Haven (New Haven, CT). Cells were cultured to $70-90 \%$ confluency in either high glucose Dulbecco's Modified Eagle Medium ((DMEM: Sigma-Aldrich, D7777) for HEK293 and WT MEF) or Minimum Essential Media ((MEM: SigmaAldrich, M0268) for WI-38). All media were supplemented with 10\% FBS (Gemini Bio, 100-106), 1\% pyruvate, and 1\% Penicillin-Streptomycin-Glutamine (PSG: Gembio, 400-110), while L-glutamine (ThermoFisher, 25030081) was additionally added to only the MEM. All cells were cultured in the presence of $5 \% \mathrm{CO}_{2}$ at $37^{\circ} \mathrm{C}$.

\subsection{Menadione Preparation}

Menadione (ThermoFisher, 58-27-5) was stored at room temperature $\left(24^{\circ} \mathrm{C}\right)$ in powder form. For all treatments involving Menadione, fresh preparation included solubilized of it in DMSO as a stock concentration of $1 \mathrm{mg} / \mathrm{ml}$ and diluted to working concentrations in either DMEM or MEM.

\subsection{Cell Viability}

HEK293 $\left(8 \times 10^{4}\right.$ cells/well $)$ and WT MEF $\left(1 \times 10^{5}\right.$ cells/well) cells were seeded in 24-well plates, while WI-38 $\left(5 \times 10^{5}\right.$ cells/well $)$ cells were plated in 48 -well plates due to their regressed cell growth to reach $70-90 \%$ confluency within 24-48 hours. Cells were then treated with various dosages of Menadione for one hour and allowed to recover in fresh media for 24 hours. Plates were then incubated with MTT $(5 \mathrm{mg} / \mathrm{ml})$ reagent for three hours, solubilized in 0.04 $\mathrm{M} \mathrm{HCl}$ solubilizing buffer, and analyzed at $570 \mathrm{~nm}$.

MTT results were then validated via Trypan Blue exclusion assay. HEK293 $\left(5 \times 10^{5}\right.$ cells/well $)$ and WT MEF $\left(3 \times 10^{5}\right.$ cells/well $)$ were seeded in $60 \mathrm{~mm}$ dishes and were grown over night. The cells were treated for one hour with 5 $\mu \mathrm{M}$ and $12.5 \mu \mathrm{M}$ of Menadione and then allowed to recover in fresh media for 24 hours. Cell viability was determined by counting the number of alive cells compared to their DMSO group via Trypan Blue. Images were taken on ZOETM Fluorescent Cell Imager at 175X.

\subsection{Evaluation of Reactive Oxygen Species Levels}

HEK293 $\left(8 \times 10^{4}\right.$ cells/well $)$ and WT MEFs $\left(1 \times 10^{5}\right.$ cells/well) were seeded in 24 well plates, while the WI-38 $\left(2 \times 10^{5}\right.$ cells/well $)$ were plated in 12 well plates. Cells were treated with $25 \mu \mathrm{M}$ of Menadione for one hour and allowed to recover for various time periods. Samples were stained with MitoSOX ${ }^{\mathrm{TM}}$ Red Mitochondrial Superoxide Indicator (ThermoFisher, M36008) as described by manufacturer's protocol 30 minutes before the end of recovery phases. Superoxide Anion species were then detected on the C6 Accuri Flow Cytometer (excitation: $488 \mathrm{~nm}$; emission: 510 $\mathrm{nm}$ in FL2). Results are displayed as the mean FL2 intensity fold change compared to the DMSO group.

\subsection{Single Cell Gel Electrophoresis Assay (SCGE, Also Known as COMET Assay)}

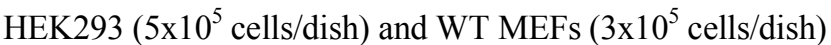
were seeded in $60 \mathrm{~mm}$ dishes the night before, while the WI$38\left(2 \times 10^{5}\right.$ cells/well $)$ were seeded in 6 well plates 24 hours prior to treatment. Cells were then treated with $25 \mu \mathrm{M}$ of Menadione for one hour and allowed to recover in fresh media for various time periods. Carefully prepared cell suspensions were then subjected to either Alkaline (Single Stranded) or Neutral (Double Stranded) COMET assay. Briefly, cells were lysed in low melting agarose in either Alkaline $(1.2 \mathrm{mM} \mathrm{NaCl}$, $0.1 \mathrm{mM}$ EDTA, $1 \mathrm{mg} / \mathrm{ml}$ of N-lauryl Sarosine, $\mathrm{pH}$ 13.2) or icecold Neutral (1X Trivegen lysis buffer, 10\% DMSO) lysis buffer and then subjected to either 1X Alkaline ( $2 \mu \mathrm{M}$ EDTA, 
$\mathrm{pH} 12.3$ ) or ice-cold $1 \mathrm{X}$ Neutral (1 mM Tris Base, $3 \mathrm{mM}$ Sodium Acetate, dropwise of Glacial acetic acid till $\mathrm{pH} 9.0$ ) washes. DNA was then separated at room temperature with 23 volts for 8 (Alkaline) or 25 minutes (Neutral) in the appropriate buffers and then precipitated with $70 \%$ ethanol (Alkaline) or in combination with DNA precipitation solution ( $0.5 \mathrm{mM}$ ammonium acetate, $47.5 \%$ ethanol) (Neutral). Slides were stained with SYBR Green and imaged on the ZOETM Fluorescent Cell Imager. The Tail Moment was calculated from 100 selected cells and analyzed via TriTrek Comet Score $^{\mathrm{TM}}$ Freeware v1.5.

\subsection{Statistical Analysis and Graphs}

Trials were conducted in triplets, and repeated three times with the average displayed and error bars representing standard error of the mean (SEM). For statistical analysis we first performed Parametric Analysis of Variance (ANOVA). The null hypothesis of ANOVA is that the location parameter (mean) of all groups (WI38, WT MEFS, and HEK293) are the same. The alternative hypothesis is that they are different. The result shows we have evidence to believe that the means are different. However, since an assumption we make when perform an ANOVA test is that the residuals are normally distributed. We checked for normality of the residual and reported the P-values of the Shapiro-Wilk test side-by-side with the ANOVA P-values. The result suggests that the residuals are not normally distributed. Thus, our ANOVA test result should be reconsidered. Toward this goal, we employ the Krustkal-Wallis test, a non-parametric version of ANOVA, to test for difference among three groups: WI38, WT MEFS, and HEK293. We also did pairwise comparison with Wilcoxon rank-sum test, a non-parametric technique to compares the location parameter (median) between two groups. The $\mathrm{R}$ script to produce statistical test results can be made available. All graphs were generated on GraphPad Prism 8.

\section{Results and Discussion}

\subsection{Cell Viability in Response to Menadione}

To assess if neuronal-like HEK293 had sensitivity to endogenous ROS, we treated them with Menadione and compared to two different fibroblast lines. Results showed that HEK293 had a significantly lower viability towards Menadione at dosages 5, 10, and $12.5 \mu \mathrm{M}$ after one hour of treatment compared to both fibroblast lines (Figure 1A). Interestingly, at $25 \mu \mathrm{M}$ the MEFs deviated from their trend and had viability similar to HEK293. This did not seem to be a general fibroblast phenotype, as the WI-38 cells did not experience that drop in viability at $25 \mu \mathrm{M}$. Comparing the $\mathrm{IC}_{50}$ values, HEK293 $\left(\mathrm{IC}_{50}=16.07 \mu \mathrm{M}\right)$ were lower than that of the WT MEFs $\left(\mathrm{IC}_{50}=22.00 \mu \mathrm{M}\right)$ and about half of the WI$38\left(\mathrm{IC}_{50}=34.08 \mu \mathrm{M}\right)$.

Menadione has been shown in the literature to disrupt basal mitochondria function. We confirmed our viability assay and conducted Trypan Blue exclusion at dosages 5 and $12.5 \mu \mathrm{M}$ for only MEFs and HEK293. Treatment with the different dosages of Menadione did not alter WT MEF morphology, while HEK29 began detaching and showed signs of blebbing (Figure 1B). Manual quantification showed that WT MEFs had a $40 \%$ and $20 \%$ increase in viability at $5 \mu \mathrm{M}$ and $12.5 \mu \mathrm{M}$ of Menadione respectively compared to their untreated group. In correlation with the MTT results, HEK293 had 75.9\% and $46.8 \%$ viability at $5 \mu \mathrm{M}$ and $12.5 \mu \mathrm{M}$ of Menadione respectively compared to their untreated groups (Figure 1C). Collectively, these results suggest that HEK293 have reduced viability when treated with Menadione.
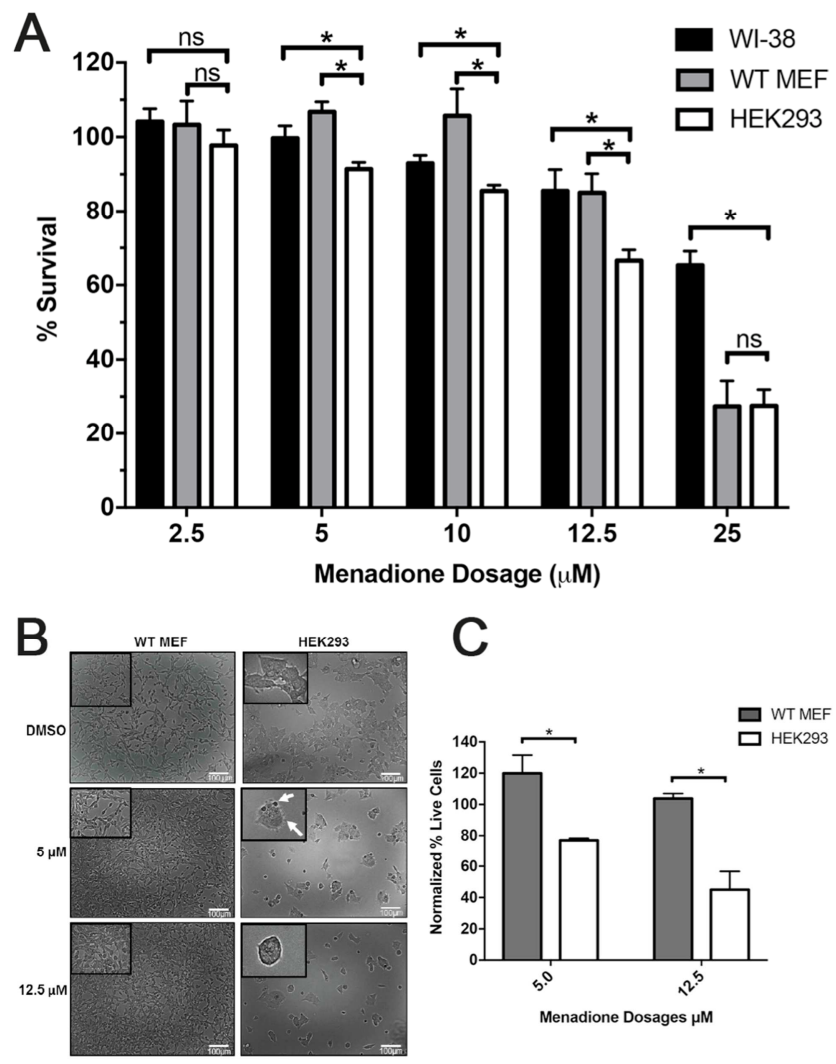

Figure 1. HEK293 cells are sensitive to Menadione treatment.

(A) Menadione induced toxicity in a dosage dependent manner as determined by MTT analysis. Cells were treated for one hour and allowed to recover for 24 in fresh media before MTT analysis. Results were normalized to the DMSO treated groups. (B) Representative Images of HEK293 and WT MEFs cells at $175 \mathrm{X}$ after two treatment points of Menadione. WT MEFs exhibited normal morphology, while HEK293 showed signs of blebbing as indicated by white arrows. (C) Cell viability was confirmed via Trypan Blue Exclusion assay. Results are expressed as the average of three independent trials with error bars representing SEM $(p<0.05$ $(*), p<0.001(* *))$. N. S. stands for not statistically significant.

\subsection{Investigating Menadione ROS Levels}

Menadione has been shown previously to up regulate SOA production. Thus, we tested if Menadione caused lethal SOA 
levels that would reduce cell survival. [17, 23]. We utilized Mitosox to assess SOA and analyzed by Flow cytometry. For this, and the remaining experiments, we used an $25 \mu \mathrm{M}$ of Menadione.

Interestingly, Menadione treatment induced some level of SOAs in all three cell types (Figure 2A). Menadione treatment caused HEK293 to produce 10 to 30 times the amount of SOA compared to WT MEFs and WI-38 respectively (Figure $2 \mathrm{~B}$ and $2 \mathrm{C}$ ). Even four hours post treatment, HEK293 still produced ten times the amount of
SOA levels compared to their vehicle group. The WT MEFs did experience a similar, but weaker, trend initially (a fold change of 3.78), but quickly sequestered their SOA levels two hours post treatment. To our surprise, the WI-38 only produced a 1.5 fold change upon initial treatment and within two hours SOA levels were reduced. It appears Menadione induced considerably different amounts of SOA among the different cell lines. And given their correlation, the highly detectable SOA could have been the driving force behind reduced viability for HEK293.

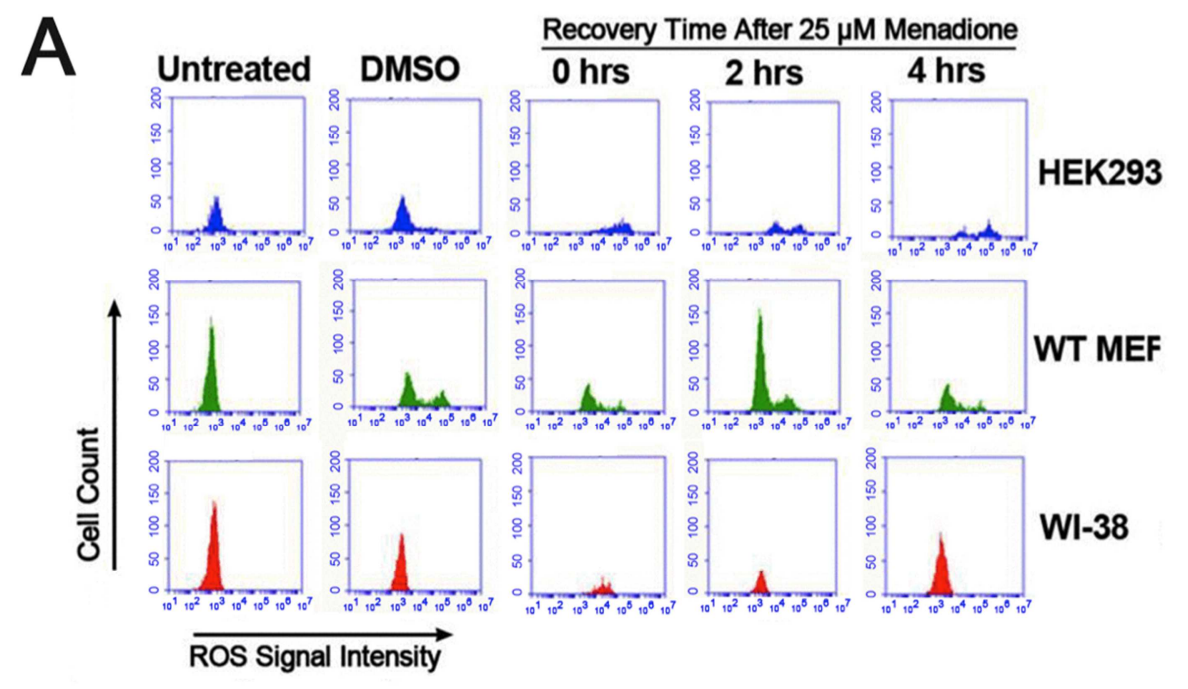

B
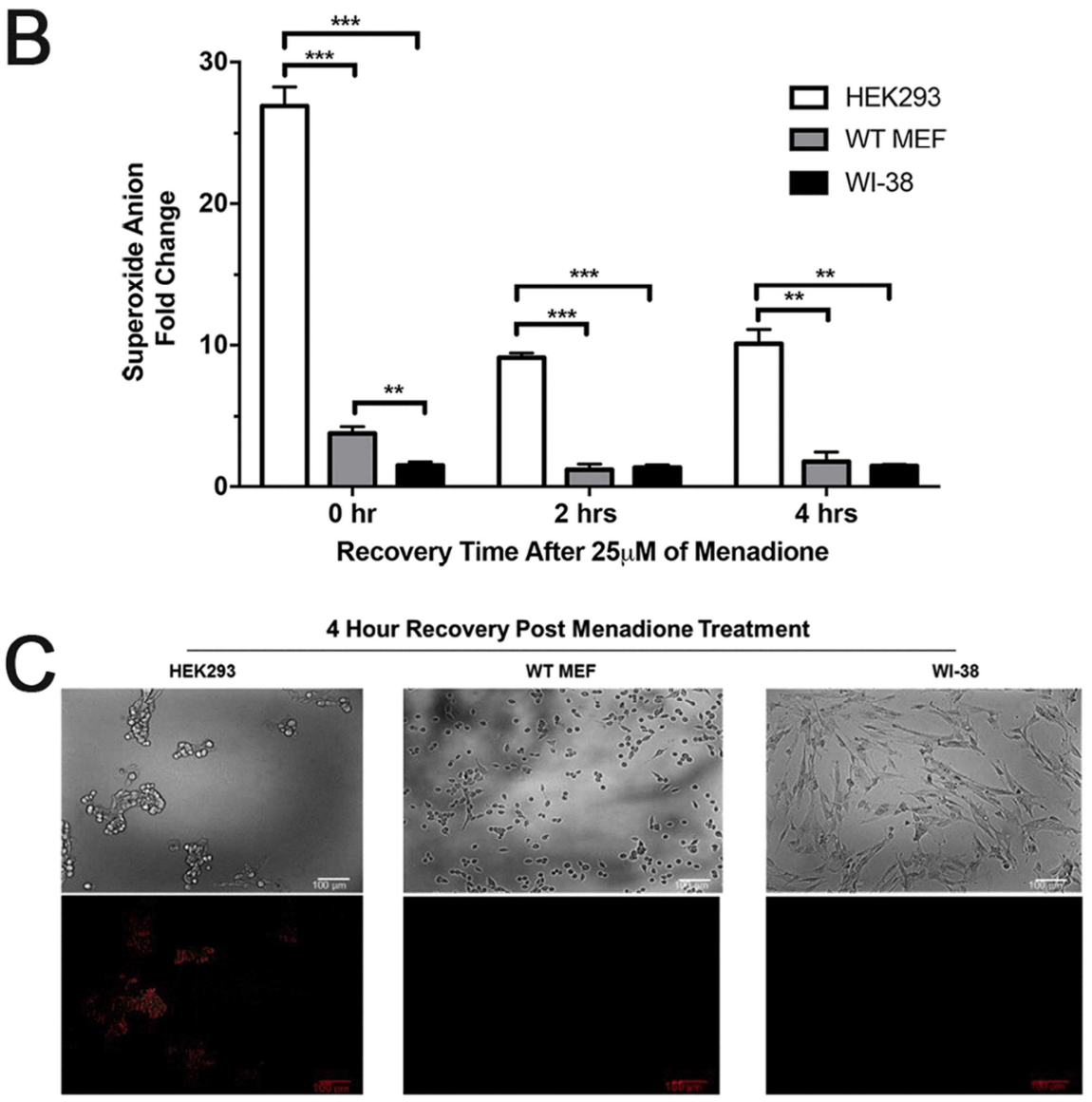

Figure 2. HEK293 cells produce high Superoxide Anions levels. 
Cells were treated for one hour, allowed to recover for various time points, and stained with Mitosox, which stains Superoxide Anions. (A) Flow cytometry fluorescence intensity distributions of Mitosox. Shifts towards the right indicate higher Superoxide Anion levels. (B) Relative FL2 fold change of Superoxide Anion production compared to the DMSO vehicle. Results are expressed as the average of three independent trials with error bars indicating SEM $(\mathrm{p}<0.001$ $(* *), \quad \mathrm{p}<0.0001(* * *))$. (C) Representative Images of Superoxide Anion production four hours post treatment. Differential interference contrast (top) and Fluorescence (bottom) were taken in identical viewing fields of the treated cells that were stained with Mitosox. Red indicates positive staining.

\subsection{Menadione Induced HEK293 Nuclear Damage and Repair}

It has been reported in the literature that Menadione produces high levels of 8-oxoG residues suggesting a role in DNA damage [24]. Since oxidized nucleotides can transgress into single stranded breaks (SSBs) and lethal double stranded breaks (DSBs), we sought to investigate if Menadione induced DNA damage. To evaluate SSBs and DSBs, we employed the usage of alkaline and neutral Single Cell Gel Electrophoresis (SCGE), commonly referred to as the COMET assay.
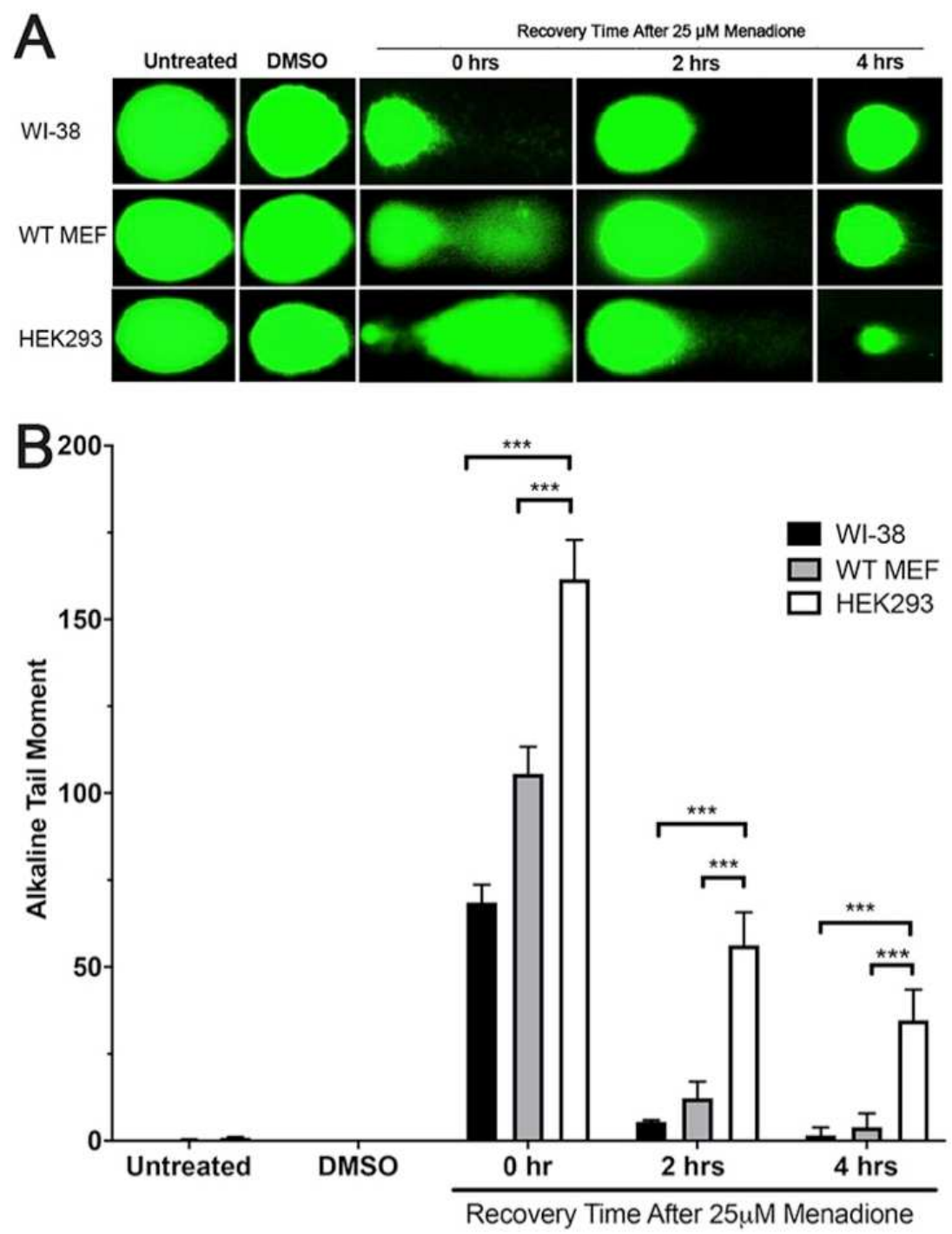

Treatment Groups

Figure 3. Reduced single stranded repair kinetics in HEK293 cells.

The results of alkaline COMET elutes that all three cell lines had DNA SSBs after exposure to $25 \mu \mathrm{M}$ of Menadione (Figure 3B and 3C). These results seemed to be in correlation with the SOA data, suggesting that the driving force behind the SSBs was the high SOA production. As expected,
HEK293 showed the most signs of damage, indicated by the presence of "hedgehog COMET," indicted by the small intact DNA and large COMET tail. Often the presence of hedgehog COMETs are a sign of extensive DNA damage or apoptosis [25]. Initial exposure to Menadione induced lower levels of 
SSBs in both WT MEFs and WI-38 compared to HEK293. Based on these results, we concluded that HEK293 experienced the most DNA damage induced by Menadione.

To investigate the repair kinetics, we treated the cells for one hour and allowed them to recover for various time periods in fresh media. Visual assessment of the COMET showed that HEK293 continued to maintain high levels of SSBs, while both WT MEFs and WI-38 had significantly lower levels of breaks (Figure 3B). Quantification of 100 randomly selected cells complemented our visual assessments (Figure 3C). We again found that HEK293 had significantly higher amounts of damage at all time points. Interestingly, we noticed that the WI-38 and WT MEFs were able to substantially repair almost all of their damage within two hours, while the HEK293 seemed to have regressed repair kinetics. Though HEK293 experienced the most initial amount of damage, they were only able to repair approximately two-thirds of their SSBs within 4 hours, whereas the two fibroblast cell lines almost completely recovered from all their damage.

Cells were treated for one hour with Menadione and allowed to recover for various time points. (A) Representative Images of Alkaline COMET data. Images were taken at $175 \mathrm{X}$ and zoomed into view. (B) Quantification of Alkaline Tail Moment. Tail Moment is a measurement of COMET tail based on the smallest detected fragmented DNA. Results are the average of 100 individual cells with error bars indicating SEM $(\mathrm{p}<0.001(* *), \mathrm{p}<0.0001(* * *))$. N. S. stands for not statically significant.

This data indicates that Menadione induced extensive DNA damage in HEK293, which was in correlation with their high SOA levels. Furthermore, alkaline COMET suggested that HEK293 had a regressed repair kinetics when allowed to recover in fresh media. Though HEK293 did have higher levels of SOA, this does not seem to be the likely cause of the regressed kinetics as the cells did continue to repair their SSBs over a 4 hour time period.
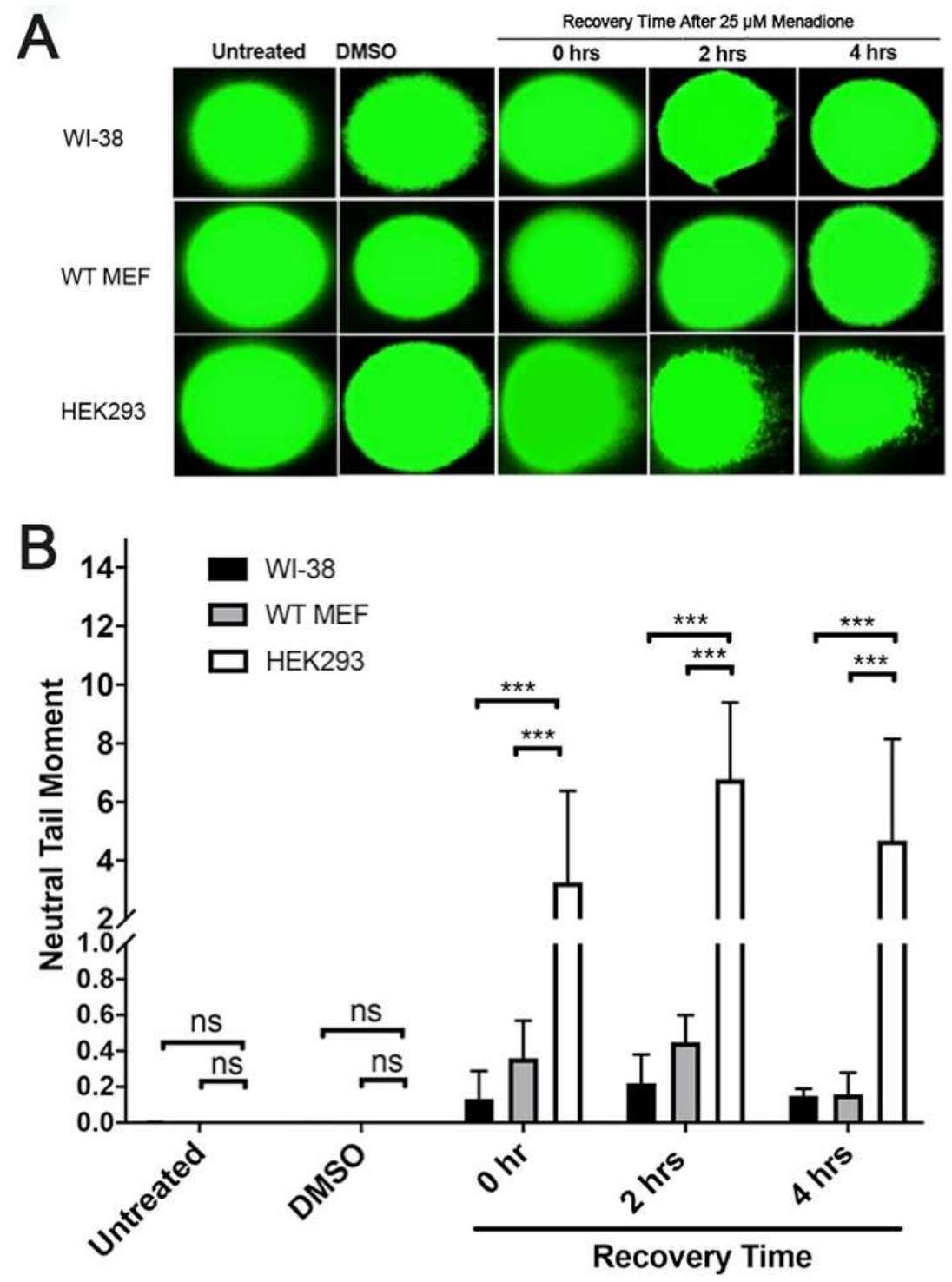

Treatment Groups

Figure 4. HEK293 cells have increased levels of double stranded breaks. 
With high detectable levels of SSBs, we next examined if these breaks became DSBs. Often SSBs have the potential to develop into DSBs due to cluster lesions of SSBs or stalling of the replication machinery. These lesions can then be eluted out via the usage of the neutral COMET (Figure 4A). Often, DSBs are normally detected at a much lower level than SSBs, due to the cell's inability to tolerate high levels of fragmentation [26]. Visual assessment of each cell line showed that only HEK293 accumulated DSBs after Menadione treatment (Figure 4B). With different periods of recovery, the DSBs became more intense and longer, while both fibroblast lines never experienced any signs of damage. Thus, it seemed that HEK293 had higher amounts of DSBs.

Cells were treated for one hour with Menadione and allowed to recover for various time points. To assess the amount of double stranded breaks present, cells were subjected to a Neutral COMET. (A) Representative Images of Neutral Data. Images were taken at $175 \mathrm{X}$ and zoomed into view. (B) Quantitative analysis of the Neutral Tail Movement. Tail Moment is a measurement of COMET tail based on the smallest detected fragmented DNA. No detectable levels of DSB were seen for the WI-38 and WT MEF cells. Results are the average of 100 individual cells with bars indicating SEM $(\mathrm{p}<0.001(* *), \mathrm{p}<0.0001(* * *))$. N. S stands for not statically significant.

Furthermore, we analyzed 100 randomly selected cells from each line to obtain an idea of the repair kinetics of DSBs. Our results relatively complemented the visual assessment, as HEK293 continued to show higher levels of DSBs with time (Figure 4C). Furthermore, both murine and human fibroblasts did not show any statistically significant levels of DSBs. It should be noted that low damage levels of the WI-38 in both the untreated and 0 hour groups are likely artifacts of the assay themselves as it was not seen at any other time periods. From the results present at this point, we concluded that HEK293 experienced lethal levels of SOA that correlated with high levels of SSBs. Additionally, due to regressed repair kinetics, those SSBs then transgressed into DSBs that ultimately lead to cellular death.

\section{Conclusions}

Excessive oxidative damage to cellular agents acts as a hallmark pathogenic phenotype of neurodegeneration [27]. Studies have proposed that major neurodegenerative diseases (e.g. Alzheimer's disease, Parkinson's disease, Amyotrophic Lateral Sclerosis) can arise due to impaired mitochondrial activity from oxidized mtDNA $[12,28]$. Interestingly, there have been several reported incidences of nuclear oxidation, but no precise mechanism of action. Our study provides some evidence of how nuclear oxidation might be linked to neurodegeneration. We found that neuron-like cells experience higher amounts of SOA when stimulated, resulting in extensive DNA damage. With higher amounts of SOA, the high amount of SSBs could then transgress into DSBs as seen in our COMET assay. The increasing amount of DSBs could then simulate apoptotic cascades and would reduce cell viability as we witnessed in our MTT and Trypan Blue exclusion assays.

In recent years, a myriad of studies have shown that the toxicity of Menadione and its route of apoptosis is tissue specific [17, 19, 23]. Here we found that both human and murine fibroblasts had higher survival rates in the presence of Menadione compared to HEK293 (Figure 3). The viability results found here is in-line with previous studies, but unique in that we witnessed reduced viability after one hour of treatment rather than 24 hours [29]. Interestingly, neuronal sensitivity towards Menadione seems to possess some level of specificity. For example, Halilovic et al. found that hCEC cells had reduced viability towards Menadione at $50 \mu \mathrm{M}$, while we saw massive cell death in HEK293 at $25 \mu \mathrm{M}$ [17]. Differences in viability might suggest that neurons and neuron-like cells responded differently to oxidative stress.

Although the WT MEFs had a similar viability as HEK293 at $25 \mu \mathrm{M}$, this is likely due to their embryonic or murine nature. Previous work has shown that MEFs upregulated their replication in the presence of low oxygen conditions (3\%), while at higher amounts $(20 \%)$ they underwent apoptosis [30]. Interestingly, we witnessed enhanced WT MEF growth at low concentrations of Menadione (i.e. $5 \mu \mathrm{M}$, $10 \mu \mathrm{M}$, and $12.5 \mu \mathrm{M}$ ), which could have created low ROS conditions that promoted growth. This seems to be a unique quality of murine fibroblasts as the WI-38 cells had an indirect relationship with Menadione concentrations.

In regards to the driving force behind the reduced HEK293 viability in response to Menadione, there seems to be a correlation with SOA production. As previously mentioned, Menadione can upregulate cytoplasmic SOA production. Though each cell line upregulated SOAs after Menadione treatment (Figure 2), HEK293 produced nearly 30 times their vehicle group and continued to maintain high levels 4 hours post treatment. The prolonged intracellular SOA exposure could be one of the driving forces behind the reduced viability, as we have witnessed in our lab that antioxidants save HEK293 from Menadione toxicity. Further validation for this theory could be seen in the WI-38, as they tolerated Menadione at higher concentrations and possessed correlating low levels of SOA. Thus, it seems that in regards to neuronal cells, it seems that the driving force behind their cell death is excessive SOA exposure.

Knowing each cell produce different level of SOA, our work primarily focused on the downstream side effects and the molecular mechanisms that govern them. We studied the toxicity of Menadione in regards to gDNA damage by utilizing both Alkaline and Neutral COMET assays. The COMET assay was used since it is considered as an excellent method due to its ability to capture small fragmentation both qualitatively and quantitatively. Our results showed that HEK293 experience higher levels of SSBs and DSBs in comparisons to murine and human fibroblasts, suggesting a different route of apoptosis then fibroblasts. Typically, SSBs are detected at a much higher concentration as they are well 
tolerated in comparison to DSBs. Our data shows that HEK293 exhibit substantial level SSBs upon Menadione exposure that some can be converted to a lethal form of DNA DSBs. The high levels of SOA that continued post treatment in HEK293 could be the likely source of the prolonged DNA damage, but this doesn't seem to be the only answer, as we did witness regressed repair kinetics. Results such as these are particularly remarkable since exogenous agents (e.g., Hydrogen Peroxide) have been shown to produce different ROS than endogenous compounds [31].

The results seen here might reflect an accurate in vivo response to oxidative stress in neurons. Therefore use of some antioxidant and nonsteroidal anti-inflammatory drugs can lower the amount of reactive oxygen species and to be among innovative chemoprevention approaches against certain human diseases [32-34].

In summary, this work provides some insight of how oxidative DNA damage induces neurodegeneration. It remains possible that as we age, we lose oxidative repair activity allowing for the extensive oxidation damage to transgress into lethal forms of DNA damage. With this information, it might suggest that loss of oxidative repair activity found early might be indicative of major diseases such as neurodegeneration.

\section{Acknowledgements}

We would like to thank the University of New Haven, NASA and Lyme Disease Association for funding. We would like to also extend our special acknowledgments to Dr. Eva Sapi and Dr. Christina Zito at University of New Haven and Dr. Joann Sweasy at Yale University for their feedback and suggestions during this study.

\section{References}

[1] Mathers, C. D., et al., Causes of international increases in older age life expectancy. Lancet, 2015. 385 (9967): p. 540-8.

[2] Brown, R. C., A. H. Lockwood, and B. R. Sonawane, Neurodegenerative diseases: an overview of environmental risk factors. Environ Health Perspect, 2005. 113 (9): p. 12506.

[3] Hampel, H., et al., The future of Alzheimer's disease: the next 10 years. Prog Neurobiol, 2011.95 (4): p. 718-28.

[4] Mayeux, R., Epidemiology of neurodegeneration. Annu Rev Neurosci, 2003. 26: p. 81-104.

[5] Przedborski, S., M. Vila, and V. Jackson-Lewis, Neurodegeneration: what is it and where are we? J Clin Invest, 2003. 111 (1): p. 3-10.

[6] Cooke, M. S., et al., Oxidative DNA damage: mechanisms, mutation, and disease. FASEB J, 2003. 17 (10): p. 1195-214.

[7] Lin, M. T. and M. F. Beal, Mitochondrial dysfunction and oxidative stress in neurodegenerative diseases. Nature, 2006. 443 (7113): p. 787-95.

[8] Ross, C. A. and M. A. Poirier, Protein aggregation and neurodegenerative disease. Nat Med, 2004. 10 Suppl: p. S107.

[9] Shukla, V., S. K. Mishra, and H. C. Pant, Oxidative stress in neurodegeneration. Adv Pharmacol Sci, 2011. 2011: p. 572634 .

[10] Andersen, J. K., Oxidative stress in neurodegeneration: cause or consequence? Nat Med, 2004. 10 Suppl: p. S18-25.

[11] Wang, H., et al., Chronic oxidative damage together with genome repair deficiency in the neurons is a double whammy for neurodegeneration: Is damage response signaling a potential therapeutic target? Mech Ageing Dev, 2017. 161 (Pt A): p. 163-176.

[12] Chen, T. S., J. P. Richie, Jr., and C. A. Lang, The effect of aging on glutathione and cysteine levels in different regions of the mouse brain. Proc Soc Exp Biol Med, 1989. 190 (4): p. 399-402.

[13] Sasaki, T., et al., Age-related changes of glutathione content, glucose transport and metabolism, and mitochondrial electron transfer function in mouse brain. Nucl Med Biol, 2001. 28 (1): p. 25-31.

[14] Martin, L. J., DNA damage and repair: relevance to mechanisms of neurodegeneration. J Neuropathol Exp Neurol, 2008. 67 (5): p. 377-87.

[15] Narciso, L., et al., The Response to Oxidative DNA Damage in Neurons: Mechanisms and Disease. Neural Plast, 2016. 2016: p. 3619274.

[16] Gandhi, S. and A. Y. Abramov, Mechanism of oxidative stress in neurodegeneration. Oxid Med Cell Longev, 2012. 2012: p. 428010 .

[17] Halilovic, A., et al., Menadione-Induced DNA Damage Leads to Mitochondrial Dysfunction and Fragmentation During Rosette Formation in Fuchs Endothelial Corneal Dystrophy. Antioxid Redox Signal, 2016. 24 (18): p. 1072-83.

[18] Iyanagi, T. and I. Yamazaki, One-electron-transfer reactions in biochemical systems. V. Difference in the mechanism of quinone reduction by the NADH dehydrogenase and the NAD (P) $\mathrm{H}$ dehydrogenase (DT-diaphorase). Biochim Biophys Acta, 1970. 216 (2): p. 282-94.

[19] Thor, H., et al., The metabolism of menadione (2-methyl-1, 4naphthoquinone) by isolated hepatocytes. A study of the implications of oxidative stress in intact cells. J Biol Chem, 1982. 257 (20): p. 12419-25.

[20] Stepanenko, A. A. and V. V. Dmitrenko, HEK293 in cell biology and cancer research: phenotype, karyotype, tumorigenicity, and stress-induced genome-phenotype evolution. Gene, 2015. 569 (2): p. 182-90.

[21] Shaw, G., et al., Preferential transformation of human neuronal cells by human adenoviruses and the origin of HEK 293 cells. FASEB J, 2002. 16 (8): p. 869-71.

[22] Thomas, P. and T. G. Smart, HEK293 cell line: a vehicle for the expression of recombinant proteins. J Pharmacol Toxicol Methods, 2005. 51 (3): p. 187-200.

[23] Loor, G., et al., Menadione triggers cell death through ROSdependent mechanisms involving PARP activation without requiring apoptosis. Free Radic Biol Med, 2010. 49 (12): p. 1925-36. 
[24] Oka, S., et al., Two distinct pathways of cell death triggered by oxidative damage to nuclear and mitochondrial DNAs. EMBO J, 2008. 27 (2): p. 421-32.

[25] Lorenzo, Y., et al., The comet assay, DNA damage, DNA repair and cytotoxicity: hedgehogs are not always dead. Mutagenesis, 2013. 28 (4): p. 427-32.

[26] van Gent, D. C., J. H. Hoeijmakers, and R. Kanaar, Chromosomal stability and the DNA double-stranded break connection. Nat Rev Genet, 2001. 2 (3): p. 196-206.

[27] Bradley-Whitman, M. A., et al., Nucleic acid oxidation: an early feature of Alzheimer's disease. J Neurochem, 2014. 128 (2): p. 294-304.

[28] Keogh, M. J. and P. F. Chinnery, Mitochondrial DNA mutations in neurodegeneration. Biochim Biophys Acta, 2015. 1847 (11): p. 1401-11.

[29] Nishiyama, T., et al., Cooperation of NAD (P) H: quinone oxidoreductase 1 and UDP-glucuronosyltransferases reduces menadione cytotoxicity in HEK293 cells. Biochem Biophys Res Commun, 2010. 394 (3): p. 459-63.

[30] Parrinello, S., et al., Oxygen sensitivity severely limits the replicative lifespan of murine fibroblasts. Nat Cell Biol, 2003. 5 (8): p. 741-7.

[31] Martins, E. A. and R. Meneghini, DNA damage and lethal effects of hydrogen peroxide and menadione in Chinese hamster cells: distinct mechanisms are involved. Free Radic Biol Med, 1990. 8 (5): p. 433-40.

[32] Kashfi, K., Anti-inflammatory agents as cancer therapeutics. Adv Pharmacol, 2009. 57: p. 31-89.

[33] Azzam, E. I., J. P. Jay-Gerin, and D. Pain, Ionizing radiationinduced metabolic oxidative stress and prolonged cell injury. Cancer Lett, 2012. 327 (1-2): p. 48-60.

[34] Hassan, G. S., Menadione. Profiles Drug Subst Excip Relat Methodol, 2013. 38: p. 227-313. 